\title{
Scrotal Exploration for Testicular Torsion and Testicular Appendage Torsion: Emergency and Reality
}

\author{
You Yu ${ }^{1}$; Feng Zhang ${ }^{2}$; Qun An ${ }^{1}$; Long Wang $^{1}$; Chao Li ${ }^{1}$; Zhilin Xu ${ }^{1, *}$ \\ ${ }^{1}$ Department of Pediatric Surgery, First Affiliated Hospital of Harbin Medical University, Harbin, China \\ 2 Department of Radiation Oncology, Second Affiliated Hospital of Harbin Medical University, Harbin, China \\ ${ }^{*}$ Corresponding author: Zhilin Xu, Department of Pediatric Surgery, First Affiliated Hospital of Harbin Medical University, Harbin 150001, China. Tel: +86-45185555139, Fax: +86- \\ 45153670428, E-mail: zhilinxucn@163.com
}

Received: March 23, 2014; Accepted: December 17, 2014

Background: Scrotal exploration is considered the procedure of choice for acute scrotum.

Objectives: We evaluated the importance of early diagnosis and testicular salvage on the therapeutic outcomes of patients with pediatric testicular torsion (TT) and testicular appendage torsion (TAT) in our geographic area.

Patients and Methods: We performed a retrospective database analysis of patients who underwent emergency surgery for TT or TAT between January 1996 and June 2009. Patient history, physical examination findings, laboratory test results, color Doppler sonography (CDS) results, and surgical findings were reviewed.

Results: A total of 65 cases were included in our analysis. Forty-two cases were followed up for at least 3 months. Testicular tenderness was identified as the major clinical manifestation of TT, while only a few patients with TAT presented with swelling. CDS was an important diagnostic modality. The orchiectomy rate was $71 \%$ in the TT group.

Conclusions: Cases of acute scrotum require attention in our area. Early diagnosis and scrotal exploration could salvage the testis or preserve normal function without the need for surgery.

Keywords: Spermatic Cord Torsion; Ultrasonography, Doppler, Transcranial; Decompression

\section{Background}

Scrotal exploration is considered the procedure of choice for acute scrotum. One cause of acute scrotal pain is testicular torsion (TT), which causes testicular hemorrhagic infarction and requires urgent care. Differential diagnosis can define TT from all other causes of scrotal pain. However, if testicular hemorrhagic infarction caused by TT persists for longer than 24 hours, the fertility function of the testis will be diminished (1), and orchiectomy is considered the last resort for nonviable testes. The clinical manifestations of TT are very similar to those of early-stage testicular appendage torsion (TAT). However, the true incidence of TAT is unknown. Therefore, the early detection of TAT could help determine whether it can be treated nonsurgically. According to clinical practice, patients with TT who are misdiagnosed with TAT could receive delayed treatment. A delay at this stage may reduce the testicular salvage rate. Although the surgical treatment of TAT is not essential, it is widely accepted since it reduces morbidity compared with conservative (nonsurgical) treatment such as anti-inflammatory analgesics and rest (2).

\section{Objectives}

In the present study, we reviewed current practices with regard to diagnosing TT and TAT and improving testicular salvage rates.

\section{Patients and Methods}

We performed a retrospective database analysis of all patients who underwent emergency surgery for TT and/ or TAT in the pediatric department of the First Affiliated Hospital of Harbin Medical University between January 1996 and June 2009. Patient history, physical examination findings, laboratory test results, color Doppler sonography (CDS) results, surgical findings, and pathological findings were reviewed for all cases. Patients diagnosed with TT or TAT by CDS or surgical findings were included in the study. Follow-up consultations were completed by telephone and outpatient clinical review. Patients with typical epididymo-orchitis (EO) were excluded since such patients do not require surgical intervention. Additional exclusion criteria were incarcerated inguinal hernia, typical scrotal trauma and hemorrhage, and testicular necrosis. This study was conducted in accordance with the declaration of Helsinki. This study was conducted with approval from the Ethics Committee of Harbin Medical University. Written informed consent was obtained from all participants. The statistical analyses were conducted using SPASS 15.0.

Copyright $@$ (2015, Growth \& Development Research Center. This is an open-access article distributed under the terms of the Creative Commons Attribution-NonCommercial 4.0 International License (http://creativecommons.org/licenses/by-nc/4.0/) which permits copy and redistribute the material just in noncommercial usages, provided the original work is properly cited. 
Yu Yet al.

\section{Results}

A total of 65 patients were identified as having undergone surgery for TT (51\%) or TAT (49\%). Forty-two patients were monitored for an average of $2.75 \mathrm{y}$ (range, 3 months to 5 years), while the other 23 were lost during follow-up. The median age of patients with TT was 11 years (range, 3 days to 16 years), while that of patients with TAT was 8 years (range, 11 months to 15 years) (Figure $1 \mathrm{~A}$ ). Three cases of TT were treated with manipulative detorsion preoperatively under ultrasonographic monitoring; one case required scrotum exploration. All other patients with TT and TAT underwent emergency surgical procedures. Two patients with TT were initially misdiagnosed as having TAT, while nine patients with TAT were misdiagnosed as having TT (Figure 1 B). The median duration of symptoms of patients with TT was 30 hours (range, 1.5-264 hours). The overall incidence of clinical findings, results of laboratory tests, and CDS evaluations are listed in Tables 1 and 2. In patients with TT, the left testicle was more frequently affected than the right testicle (64\% vs 36\%, respectively). All patients with TT except for a 3-day-old infant with extravaginal torsion were classified as having intravaginal torsion. The median degree of torsion in patients with TT was $450^{\circ}$ (range, $90-1080^{\circ}$ ). The testicle affected by TT was considered nonviable and removed at the time of exploration in 22 (71\%) cases. In the other nine patients, the testis was considered viable; testicular incision decompression (TID) surgery was performed in three, while the testis was preserved in six. All patients underwent simultaneous fixation of the contralateral testis to prevent bilateral torsion.

In one case, a 5-year-old boy suffered sudden right testicular pain for approximately 30 minutes and was admitted to the emergency room. The patient was subsequently diagnosed with right-sided TT and treated with manipulative detorsion. Following treatment, normal testicular blood flow resumed as indicated by color Doppler flow imaging. However, the patient complained of pain 1 hour later and scrotal exploration surgery was performed. Bilateral bell clapper syndrome was confirmed during surgery, which also indicated that the right testis had undergone $120^{\circ}$ clockwise torsion. Bilateral testicular orchiopexy surgery was then performed. In another case, a 2-year-old boy presented with sudden testicular pain that lasted for approximately 24 hours. CDS revealed decreased blood flow to the testis. Scrotal exploration was performed; however, the misdiagnosis of TT was confirmed. On the first postoperative day, the patient developed a Pseudomonas aeruginosa infection that was detected by urine culture. The patient received carbenicillin sodium injections for 5 days, and no subsequent clinical signs or lab findings were detected.

Three patients suffered testicular pain lasting at least 12 hours before presenting to the hospital. TID surgeries were performed on all three patients, and the degree of torsion was $270-360^{\circ}$ at the time of exploration. The af- fected testes presented with swelling and bruising, but they were not considered cyanotic. Even after resetting, the surface of the testes still appeared to be bruised; therefore, an incision surgery was performed to improve the testicular blood flow. When the bruising plague scattered on the surface, we injected $2 \mathrm{~mL}$ of $1 \%$ lidocaine into the testis and observed the tissue color changes for 3-5 minutes. The tunica albuginea was closed with 5-0 absorbable sutures after confirmation that the testis had returned to its normal color. Testicular appendage resection was performed on all patients with TAT. Scrotal exploration was performed in three patients suspected of having TAT. Two of these patients presented with epididymal cysts, while the other patient presented with epididymal nodules. These patients underwent cyst/nodule resection and were not included in the statistical analysis of patients with TAT.

Nearly all of the patients experienced pain relief after surgery. Three patients who underwent TID developed postoperative scrotal swelling, which resolved gradually after 3-5 days and did not require additional specialized care. One patient experienced testicular atrophy 1 month after surgery. These patients did not exhibit further clinical signs at the 6-month follow-up. No other postoperative complications were noted over the subsequent 5 years period. Pathological examinations were performed on all excised testicles. A rare precursor B lymphoblastic testicular tumor was detected in a patient with TT. All other patients with TT showed hemorrhagic necrosis.

Table 1. Clinical Findings and Results of Laboratory Tests on Boys With testicular Torsion (TT), and Testicular Appendage Torsion (TAT) ${ }^{\mathrm{a}, \mathrm{b}}$

\begin{tabular}{lcc}
\hline $\begin{array}{l}\text { Clinical Findings and Laboratory } \\
\text { Tests }\end{array}$ & TT $(\mathbf{n}=\mathbf{3 3})$ & TAT $(\mathbf{n}=\mathbf{3 2})$ \\
\hline Duration of symptoms $<\mathbf{6} \mathbf{h}$ & $3(9)$ & $7(22)$ \\
\hline $\begin{array}{l}\text { Duration of symptoms } \mathbf{6 - 2 4} \mathbf{~ h} \\
\text { Duration of symptoms }>\mathbf{2 4 h}\end{array}$ & $1(3)$ & $9(28)$ \\
\hline Pain/Tenderness & $27(82)$ & $32(100)$ \\
\hline Swelling & $19(58)$ & $4(13)$ \\
Nausea/Vomiting & $18(55)$ & $6(19)$ \\
\hline Absence of cremasteric reflex & $17(52)$ & $2(6)$ \\
\hline Positive Prehn sign & $15(45)$ & $5(16)$ \\
\hline Erythema & $11(33)$ & $1(3)$ \\
\hline Abnormal testicle direction & $9(27)$ & - \\
\hline Fever $\left(>\mathbf{3 8}{ }^{\circ} \mathbf{C}\right)$ & $7(21)$ & $1(3)$ \\
CBC WBC $>\mathbf{1 0} \mathbf{9} / \mathbf{L}$ & $7(21)$ & $2(6)$ \\
CRE $>\mathbf{5} \mathbf{~} \mathbf{m g} / \mathbf{L}$ & $21(64)$ & $4(13)$ \\
\hline
\end{tabular}

a Abbreviations: CBC, complete blood count; CRE, creatinine; TAT, testicular appendage torsion; TT, testicular torsion; WBC, white blood cell.

b Data are presented as No.(\%). 

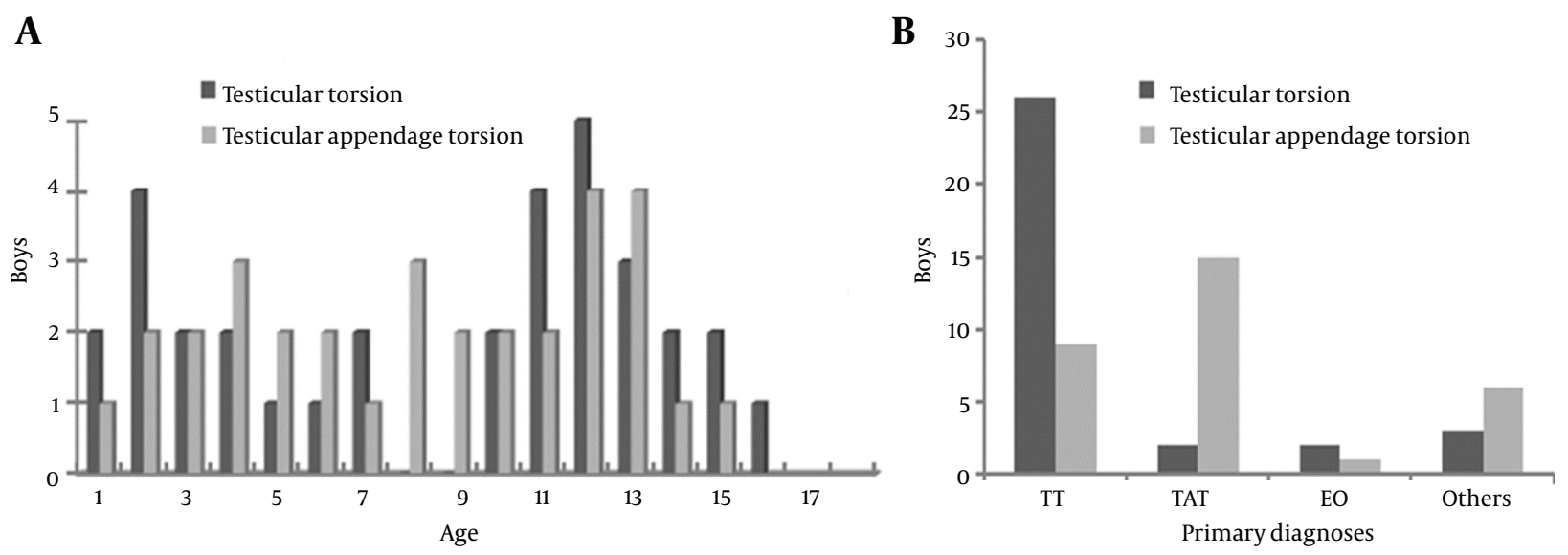

Figure 1. A) The distribution of patients in different ages with TT and TAT. B) The number of patients with primary diagnoses within TT and TAT.

Table 2. Color Doppler Ultrasound (CDS) Evaluation in Boys With Testicular Torsion (TT), and Testicular Appendage Torsion $(\mathrm{TAT})^{\mathrm{a}, \mathrm{b}}$

\begin{tabular}{|c|c|c|}
\hline CDS & $T T(n=33)$ & TAT $(n=32)$ \\
\hline Hydrocele & $29(88)$ & $11(33)$ \\
\hline Abnomal testicular texture & $28(85)$ & $1(3)$ \\
\hline Swollen testis & $25(76)$ & $2(6)$ \\
\hline Scrotal wall edema & $23(70)$ & $5(16)$ \\
\hline Hematoma & $5(15)$ & $1(3)$ \\
\hline Enlarged epididymis & $5(15)$ & $3(9)$ \\
\hline Extra testicular nodule & $3(9)$ & $29(91)$ \\
\hline \multicolumn{3}{|l|}{ Blood flow of testis } \\
\hline Normal & $1(3)$ & $29(91)$ \\
\hline Increased & 0 & $2(6)$ \\
\hline Decreased or absent & $32(97)$ & 0 \\
\hline
\end{tabular}

\section{Discussion}

Scrotal problem in pediatric patients is a pathology that presents with scrotal pain, erythema, swelling, hydroceles, and edema. It is commonly caused by TAT, incarcerated scrotum, TT, and EO and occasionally by trauma, hemorrhage, testicular malignancy, and idiopathic scrotal edema (3). Experienced pediatric surgeons can identify the cause of scrotal pathology by taking a clinical history and performing a clinical examination (4). However, differentiation between TT and TAT causes a dilemma in the early stages of the disease. Based on our experience, scrotal tenderness, cremasteric reflex, and erythema are useful diagnostic signs of TT and TAT. We believe that scrotal exploration is a safe procedure when performed with the knowledge of an accurate diagnosis.
The necessity for early treatment of TT to avoid testicular infarction is well recognized $(5,6)$. TT reduces blood supply to the testis, which subsequently leads to hemorrhage, infarction, and necrosis. Many studies have shown that testicular infarction begins within the first 2 hours of TT onset, irreversible damage occurs after 6 hours, complete infarction develops after 24 hours, and fertility function is inevitably diminished thereafter (1, 7). Statistical analysis indicates that aggressive treatment performed within 6 h of TT onset has a 93\% testicular salvage rate. In contrast, only $10 \%$ of cases are resolved after 24 hours (8). Of the patient cases presented here, 22 (22/31, $71 \%$ ) underwent orchiectomy surgery even though the treatment initiation was not delayed. This finding can be explained by delays in seeking medical attention. Inadequate care offered by less experienced first line pediatric surgeons is the major cause in most cases.

A literature review suggests that the incidence of TAT is quite different from that of TT $(9,10)$ and that the incidence of TAT could, in theory, be higher than that of TT (11). In our investigation, TAT accounted for $49 \%$ (32/65) of cases. This value is considered lower than actual rates and may be explained by an unwillingness to visit a doctor's office or the pain automatically subsiding after a few days. TAT commonly leads to appendage swelling, necrosis, and finally absorption. Surgical intervention in cases of TAT may not be necessary. For patients diagnosed with TT, surgical scrotal exploration can offer a differential diagnosis, thus minimizing the risk of testicular loss through misdiagnosis (12). However, treatment approaches are constantly debated. Even medical professionals advocating for conservative management acknowledged that $22 \%$ of cases require urgent explorative surgery for differential diagnosis and that $14 \%$ of patients receiving conservative management develop persistent pain, which requires testicular excision (13). Of the cases reviewed in our analysis, two patients with a primary diagnosis of TAT were confirmed as having TT. Therefore, any risks 
of avoiding testicular loss are not wasted. On the other hand, scrotal exploration and excision of the torted appendage under general anesthetic is a safe procedure with minimal morbidity (9). This enables an accurate diagnosis, relieves symptoms, and alleviates the anxiety of a patient's parents.

CDS is considered the primary imaging modality for the evaluation of diverse etiologies. Reported diagnostic performance values for CDS of TT were $69-86 \%$ sensitive, $87-100 \%$ specific, and $73-97 \%$ accurate. CDS has a reported positive predictive value of $100 \%$ and negative predictive value of $97.5 \%$ (14-16). Furthermore, CDS together with clinical evaluations is a reliable technique for the identification of TT (7). In our reviewed cases, patients with acute scrotum were transferred immediately and scrotal CDS examinations were performed without delay. This proved to be a reliable initial strategy for minimizing testicular loss. Moreover, Soccorso et al. (17) suggest that scrotal CDS should not be used as an emergency investigation, while surgical exploration of the scrotum in patients experiencing pain must be performed urgently without a preoperative morphologic assessment.

Orchiectomy rates vary widely in the literature and are $39-71 \%$ in most series (18-20). The orchiectomy rate in our analysis was higher than the published values. The major cause for the high orchiectomy rate is considered to be the inherent delay in seeking early care. The median duration of symptoms experienced by patients included in our analysis was 30 hours, while the median degree of torsion was $450^{\circ}$. The results suggest that factors that cause treatment delays are critical for patients with torsion. TT is a common source of litigation in urological practice $(21,22)$. The most common reasons for litigation tend to be delayed diagnosis, missed diagnosis, and treatment delays. In our geographical region, more multicenter collaborative efforts are needed with rural hospitals to improve diagnostic accuracy. Cases of acute scrotum, particularly TT, should be accurately diagnosed. Testicular function, and the testis itself, can only be preserved or salvaged in patients who receive a differential diagnosis and undergo surgical procedures without delay.

\section{References}

1. Schutte B, Becker H, Vydra G. [Exocrine and endocrine testicular function following unilateral torsion--a retrospective clinical study of 36 patients]. Urologe A. 1986;25(3):142-6.

2. Makela E, Lahdes-Vasama T, Rajakorpi H, Wikstrom S. A 19-year review of paediatric patients with acute scrotum. Scand J Surg.
2007;96(1):62-6.

3. Halb C, Eschard C, Lefebvre F, Brunel D, Abely M, Bernard P. [Acute idiopathic scrotal oedema in young boys: A report of ten cases and a review of the literature]. Ann Dermatol Venereol. 2010;137(12):775-81.

4. Murphy FL, Fletcher L, Pease P. Early scrotal exploration in all cases is the investigation and intervention of choice in the acute paediatric scrotum. Pediatr Surg Int. 2006;22(5):413-6.

5. Mushtaq I, Fung M, Glasson MJ. Retrospective review of paediatric patients with acute scrotum. ANZ J Surg. 2003;73(1-2):55-8.

6. Khaleghnejad-Tabari A, Mirshermirani A, Rouzrokh M, Mahmudi M, Baghaiepour MR, Ghaffari P, Hatamian B, et al. Early exploration in the management of acute scrotum in children. Iran J Pediatr. 2010;20(4):466-70.

7. Cattolica EV, Karol JB, Rankin KN, Klein RS. High testicular salvage rate in torsion of the spermatic cord. J Urol.1982;128(1):66-8.

8. Waldert M, Klatte T, Schmidbauer J, Remzi M, Lackner J, Marberger M. Color Doppler sonography reliably identifies testicular torsion in boys. Urology. 2010;75(5):1170-4.

9. Lewis AG, Bukowski TP, Jarvis PD, Wacksman J, Sheldon CA. Evaluation of acute scrotum in the emergency department. J Pediatr Surg. 1995;30(2):277-81.

10. Molokwu CN, Somani BK, Goodman CM. Outcomes of scrotal exploration for acute scrotal pain suspicious of testicular torsion: a consecutive case series of 173 patients. BJU Int. 2011;107(6):990-3.

11. Holland JM, Graham JB, Ignatoff JM. Conservative management of twisted testicular appendages. J Urol.1981;125(2):213-4.

12. Sandor L, Gajda T, Aranyi V, Csizy I, Cserni T. [Role of ultrasonography in the urgent differential diagnosis of acute scrotum]. Orv Hetil. 2011;152(23):909-12.

13. Lam WW, Yap TL, Jacobsen AS, Teo HJ. Colour Doppler ultrasonog raphy replacing surgical exploration for acute scrotum: myth or reality? Pediatr Radiol. 2005;35(6):597-600.

14. Burks DD, Markey BJ, Burkhard TK, Balsara ZN, Haluszka MM, Can ning DA. Suspected testicular torsion and ischemia: evaluation with color Doppler sonography. Radiology. 1990;175(3):815-21.

15. Zini L, Mouton D, Leroy X, Valtille P, Villers A, Lemaitre L, Biserte J, et al. [Should scrotal ultrasound be discouraged in cases of suspected spermatic cord torsion?]. Prog Urol. 2003;13(3):440-4.

16. Liang T, Metcalfe P, Sevcik W, Noga M. Retrospective review of diagnosis and treatment in children presenting to the pediatric department with acute scrotum. AJR Am J Roentgenol. 2013;200(5):W444-9.

17. Soccorso G, Ninan GK, Rajimwale A, Nour S. Acute scrotum: is scrotal exploration the best management? Eur J Pediatr Surg. 2010;20(5):312-5.

18. Baker LA, Sigman D, Mathews RI, Benson J, Docimo SG. An analysis of clinical outcomes using color doppler testicular ultrasound for testicular torsion. Pediatrics. 2000;105(3 Pt1):604-7.

19. Kaye JD, Shapiro EY, Levitt SB, Friedman SC, Gitlin J, Freyle J, Palm er LS, et al. Parenchymal echo texture predicts testicular salvage after torsion: potential impact on the need for emergent exploration. J Urol. 2008;180(4 Suppl):1733-6.

20. Boettcher M, Bergholz R, Krebs TF, Wenke K, Aronson DC. Clinical predictors of testicular torsion in children. Urology. 2012;79(3):670-4

21. Hsieh MH, Tan AG, Meng MV. Medical malpractice in American urology: 22-year national review of the impact of caps and implications for contemporary practice. J Urol. 2008;179(5):1944-9.

22. Mellick LB. Torsion of the testicle: it is time to stop tossing the dice. Pediatr Emerg Care. 2012;28(1):80-6. 